\title{
The Identification of Neurons Research
}

\author{
Xiaojing Shang \\ Electrical and Information Engineering Department \\ The City College Of Jilin Architectural And Civil Engineering Institute, Changchun, 130000, China \\ (e-mail: 312511605@qq.com).
}

\begin{abstract}
In view of the present medical neurons characteristic cognition and human brain plan in the neurons of the limitation of recognition, this paper puts forward the neurons identification method. First the $L$ - Measure software to neuron geometry feature extraction, and then to extract high dimensional feature through the principal component analysis dimension reduction processing. Combined classifier with pyramidal neurons, general Ken wild neurons, motor neuron, sensory neurons, double neurons, level 3 neurons and multistage neurons 7 kinds of neurons are classified. Experimental results prove that the probabilistic neural network, the BP neural network, fuzzy classifier composed of classifier recognition effect is superior to the arbitrary single classifier.
\end{abstract}

Keywords- neurons; L - Measure; Principal component analysis; Combined classifier, Recognition and classification

\section{PREFACE}

The brain is in biological structure and function of the most complex organization, including a billion of nerve cells (neurons)[1]. Neurons space geometry research is the human brain plan an important project. The understanding of the characteristics of neurons, the most basic problem is the classification of neurons.

At present, the simple classification of neurons is mainly: (1) according to the protuberant how many can be divided into multi polar neuron neurons; Bipolar neurons and single neuron. (2) According to the function of neurons and points is given priority to neurons, sensory neuron, motor neuron and interneuron's, etc. Main neuron's main function is output neural circuit information. [2-3].

How to identify distinguish between different types of neurons, the problem at present is still not solved in science.

This paper using L - Measure software [4] accurate comprehensive extraction neuron geometry features. Using principal component analysis to these characteristics of dimension reduction processing, reserve classification ability characteristics.

The BP neural network is more perfect and the most common classifier, but in the process of training is easy to fall into the local minimum point, long training time and probability neural network training time is short, the structure is stable, not easy to fall into the local minimum value can make up for the shortcomings of BP network. These two kinds of network although classification effect is good, but for features overlap serious sample classification result is bad, and fuzzy logic classifier is suitable for data overlapping serious sample classification. Therefore, the three classifier combination, make up this deficiency, adopt the "vote" choice method to pyramidal neurons, general Ken

wild neurons, motor neuron, sensory neurons, double neurons, level 3 neurons and multistage neurons 7 kinds of neurons are classified.

\section{NEURONS OF FEATURE EXTRACTION AND FEATURE SELECTION}

In the neurons in the process of recognition to select feature is very important, if use features not appropriate or not fully, can reduce the recognition accuracy. Therefore, the introduction of characteristics pass through from a limited amount, and from more to less process.

Figure 1 is 7 kinds of different neuron geometry graph. From figure 1 can see, different types of neurons geometry difference is obvious.

Table 1 is all kinds of neuron corresponding space form data. From table 1 can also see, categories of data between difference obvious, especially the sensory neurons cell surface area and dry number and other kinds of neurons have significant difference.

Feature extraction is the principle of simple calculation and separation between data of large data as sample characteristics. Therefore, based on the geometry of neurons classification has certain advantage.

This paper using L - Measure software to neuron geometry feature extraction. Due to the extraction of feature dimension too much, so through the principal component analysis method to extract the feature of the dimension reduction processing, to simplify the calculation process.

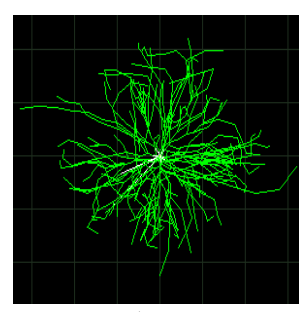

a.motor neuron

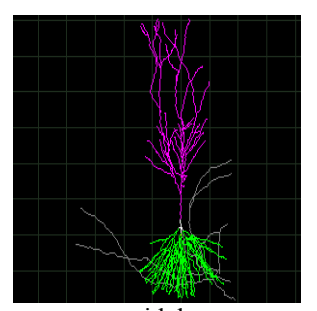

c.pyramidal neurony

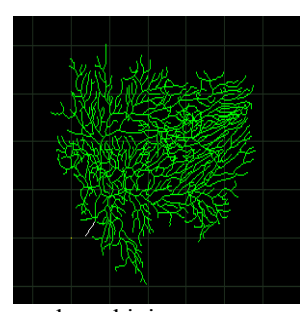

b.purkinje neuron

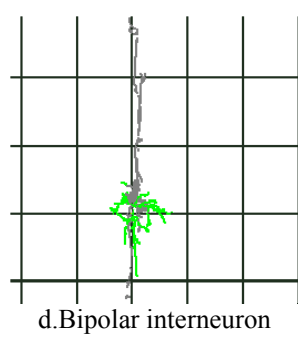




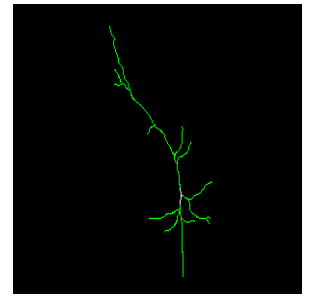

e.Tripolar interneuron

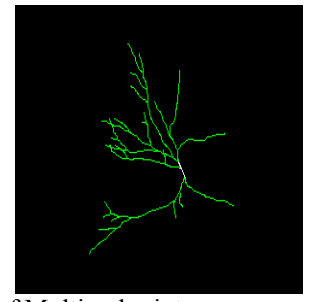

f.Multi polar interneuron

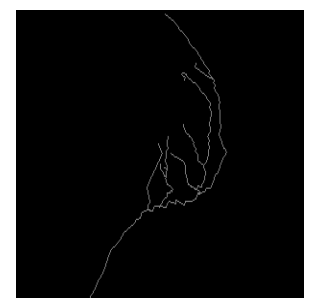

g.sensory neuron

Figure 1. morphological figures of 7 kinds neurons

TABLE I. SPATIAL DATA FOR 7 KINDS NEURONS

\begin{tabular}{|c|c|c|c|c|c|c|}
\hline $\begin{array}{c}\text { Spatial } \\
\text { charac- } \\
\text { teristics }\end{array}$ & $\begin{array}{c}\text { Spatial } \\
\text { charac- } \\
\text { teristics }\end{array}$ & $\begin{array}{c}\text { The } \\
\text { number } \\
\text { of dry }\end{array}$ & $\begin{array}{c}\text { Bifurcati } \\
\text { on } \\
\text { number }\end{array}$ & $\begin{array}{c}\text { The } \\
\text { number } \\
\text { of } \\
\text { branches }\end{array}$ & Wide & High \\
\hline $\begin{array}{c}\text { motor } \\
\text { neurons }\end{array}$ & 29910.9 & 15 & 151 & 331 & 2541 & 1922 \\
\hline $\begin{array}{c}\text { purkinje } \\
\text { neuron }\end{array}$ & 511.087 & 1 & 343 & 688 & $\begin{array}{c}120.8 \\
2\end{array}$ & $\begin{array}{c}105 . \\
78\end{array}$ \\
\hline $\begin{array}{c}\text { Pyramid } \\
\text {-dal neu } \\
\text { rons }\end{array}$ & 610.356 & 6 & 87 & 185 & 232.5 & $\begin{array}{c}715 . \\
5\end{array}$ \\
\hline $\begin{array}{c}\text { Bipolar } \\
\text { interneu } \\
\text { ron }\end{array}$ & 426.754 & 5 & 61 & 131 & $\begin{array}{c}233.3 \\
1\end{array}$ & $\begin{array}{c}299 . \\
61\end{array}$ \\
\hline $\begin{array}{c}\text { tripolar } \\
\text { interneu } \\
\text { ron }\end{array}$ & 1318.45 & 3 & 10 & 25 & $\begin{array}{c}180.3 \\
3\end{array}$ & $\begin{array}{c}358 . \\
36\end{array}$ \\
\hline $\begin{array}{c}\text { Multipo } \\
\text {-lar } \\
\text { interne- } \\
\text { uron }\end{array}$ & 1976.34 & 5 & 20 & 49 & 274.8 & 331. \\
\hline $\begin{array}{c}\text { sensory } \\
\text { neurons }\end{array}$ & 0 & 0 & 11 & 23 & 107.0 & 138. \\
\hline
\end{tabular}

\section{A. Feature extraction}

L - Measure software is calculation neurons software, can accurate comprehensive calculated neuron geometry features. In this paper, through in the related website [5] download neurons data by using the software extracted neuron cell surface area, the number of dry, branch number, number of branch, width, height, depth, diameter, length, area, volume, trunk taper, branch power law, branch Angle of 20 major characteristics.

\section{B. Principal component analyses}

Principal component analysis [6] is the original variable reset to a set of new independent of each other a few variables, at the same time, according to actual needs, from which we can take out a few less comprehensive variables as much as possible to reflect the original variable information statistics method, mathematics is the processing dimension reduction of a kind of method.

USES the original each feature to structure a number of new features, each feature is the original characteristics of the function, but the new characteristics of the total should be less than the total original features. In this way, a new geometric feature not only retains the original characteristics of the main information, and to reduce the feature dimension which reduce space dimension of purpose. 20 features for classification will increase the complexity of the calculation, so, using the method of principal component analysis to these 20 characteristics was analyzed, and reduces the dimension.

Through the principal component analysis after, and the 20 features simplified as four major geometric feature.

\section{THE CLASSIFICATION OF NEURONS}

In this paper the classification method is in the three basic classification method of BP neural network, probabilistic neural network and fuzzy logic classifier for improvement. According to three kind of classifier their respective advantages and disadvantages, the three classifier combination together on their classification results "vote" on the classification of neurons.

\section{A. The BP neural network}

$\mathrm{BP}$ algorithm is a kind of commonly used neural network algorithm; it is a kind of supervised learning algorithm. The purpose of the study is to use the network output and the error between the expected output to modify their weights, make the actual and expectations to as much as possible, even if the network output layer error sum of squares minimum, through continuously in relative to the error function slope down direction calculation of network weights and bias change and gradually approaching target..

BP neural network is generally divided into three layers: input layer, hidden layer and output layer [7].

\section{B. Probability neural network}

Probabilistic neural network is put forward in 1990 by Specht [8] a radial basis function (RBF) network important deformation [9]. Based on the statistics of the principle of neural network model in the classification function and optimal bays classifier equivalent, it USES multivariate Parzen Windows estimate inhomogeneous probability density function and the Bayesian posterior probability as the output, and the traditional multilayer feed forward network, the training time is short, the structure is stable, has strong nonlinear recognition ability, and not easy to converge to a local minimum point, to make up for the deficiency of BP neural network, especially suitable for solving pattern recognition problems.

Probabilistic neural network consists of three layer forward network composition, input layer by the source node is composed; the second is hidden layer, the number of units by the problems described and decides; the third is the output layer. Output layer probability maximum category buy 1 , the other set 0 . 


\section{Fuzzy logic [10-11]}

The BP neural networks and probabilistic neural networks are more mature network, for most of the sample classification problem can be very good solution, but to feature data overlapping serious sample, classification effect is not very good. And fuzzy logic can be a very good solves this problem, fuzzy logic classifier for overlap more characteristic data classification effect is very good.

Fuzzy logic mainly through the establishment of the membership function to classification, the principal component analysis to get four characteristics into a collection, and from every feature set find maximum and minimum value as the set range, then every feature of the data range is divided into is interval, so each characteristics respectively have is interval.

\section{Combination "casts a ballot "}

BP neural networks is easy to be caught in part external point, train time too long; Probability neural networks training speed is quick, can not be caught in part external point. Blurred logic classification implement is kind with graver overlapping data characteristic classification effect. Carrying out the basic classification of nerve according to the three kinds respective excellent classification implement shortcoming, the method them being formed congregating together, covering the shortage, adopt three kinds classification implement to cast a ballot.

Among them the meanest is being casts a ballot regulation. Seeing the name of a thing one thinks of its function, three kinds classification implement carry out casting a ballot classifies results. The acquisition ticket counts the result that the most classification result is in order being victorious finally. First, training and testing effect checking different kind nerve basic respectively according to BP neural networks, probability neural networks and blurred logic classification implement interpose the nerve basic of all kinds weight value under different classification implement. At the same time, to the classification between three kinds classification implement [12-15].

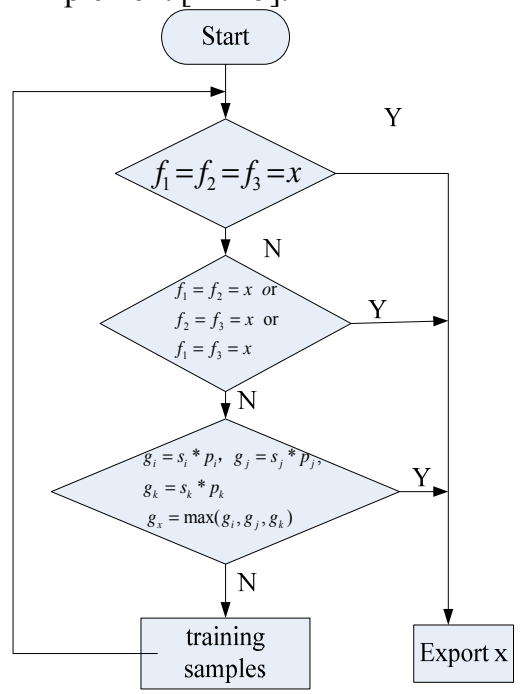

Figure 2. voting rules flowchart

\section{SIMULATED EXPERIMENT AND RESULT ANALYSIS}

This paper adopts the L-Measure software to calculate the basic geometric nerve characteristic, adopt the host be accomplished analysis to decrease geometric 20 form characteristics to 4 characteristics and, nerve basic choice per kind 20 group characteristics carry out the neural networks training, adopt BP finally on three kinds classification implement difference, probability neural networks and blurred logic classification implement constitute but able classification implement carries out a testing on 35 nerve basic.

From the repeated experiment, and among 2.3, weight value sets up method, the weight value difference ascertaining three kinds classification implement is 0.333 , $0.345,0.322$, as well as nerve of all kinds weight of worth is $p_{\mathrm{i}}, p_{\mathrm{j}}, p_{\mathrm{k}}, 1 \leq \mathrm{i}, \mathrm{j}, \mathrm{k} \leq 7$. The method adopt three kinds classification implement to cast a ballot classifies and distinguishes to 35 nerve basic, the experiment tests effect as shown the Figure 3.

From the figure, we can see the 35 nerve basic only when 3 nerve basic there exists error in actual category and forecast category in secondary picture, the method identification effect adopt three kinds classification implement to cast a ballot is fairly good. Table 2 is that various classification implement recognition rate is parallel.

From the table 2 can perceive the all essential recognition rate height constituting three kinds classification implement adopt the method casting a ballot to carry out the implement distinguishing it's rightness recognition rate than one kind classifies arbitrarily on nerve basic .

But, this two kinds networks pile up grave sample book classification rightness recognition rate low to characteristic data, the characteristic of blurred logic classification implement comparatively suitable data piles up but grave sample book classification, because blurred logic classification implement can wait for a fraction to come to change recognition rate by adjustment, counteract starting from when that the number piling up grave waiting waits for a number right away increasingly much, may also check the experiment bear fruit very excessively. This paper through repeated tests to determine when the equal number of 10 , the best result for classification.

Classification implement combines with the three kinds, the weight setting up every classification implement and nerve basic of all kinds is worth , casting a ballot carrying out classification result's chooses. Demonstration, that method have obvious rise to basic nerve classification recognition rate, being method's turn has effect. 


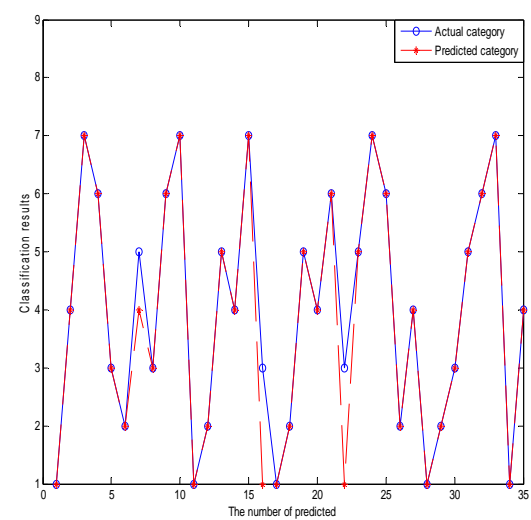

Figure 3. classification test results graph

TABLE II. COMPARISON OF RECOGNITION RATE OF EACH CLASSIFIER

\begin{tabular}{|c|c|c|c|c|}
\hline Classifier & $\begin{array}{c}\text { BP neural } \\
\text { network } \\
\text { BP }\end{array}$ & $\begin{array}{c}\text { Probabilis } \\
\text { tic neural } \\
\text { network }\end{array}$ & $\begin{array}{c}\text { Combinatio } \\
\text { n of } \\
\text { classification }\end{array}$ & Mix Vote \\
\hline $\begin{array}{c}\text { Recognition } \\
\text { rate }\end{array}$ & $82.8 \%$ & $85.7 \%$ & $77 \%$ & $91.4 \%$ \\
\hline
\end{tabular}

V. CONCLUSION

This paper based on the geometric morphology of neurons, with the help of L-Measure software, complete and accurate extraction of neurons of the 20 geometric shape, because the characteristics of too many dimensions to calculation of impact, using principal component analysis to feature dimension reduction treatment to obtain 4 main characteristics.

Because the BP neural network in the classification process is easy to fall into local extreme point, long training time; Probability neural networks training speed is quick, can not be caught in part external point; A fuzzy logic classifier on the data overlapping more serious features good classification effect. Entering looking on 4 kinds characteristics as BP neural networks, probability neural networks and combined blurred logic classification implement three kinds classification implement classification implement, adopt unity to cast a ballot regulation other nerve basic carries out identification classification on cone nerve basic, Puken open space nerve basic, 7 move nerve basic, sensory neuron, pair of level nerve basic, three-level nerve basic and multi-level nerve basic kind. Experiments prove that the combined classifiers, after correct recognition rate than either a classifier recognition rate are high.

Average classification method recognition rate brought forward by the main body of a book can reach $91.4 \%$, But the nerve basic classification effect to part kind is fairly not good enough. Classification of short time, high accurate identification rate, more efficient classification method still remain at further research.

\section{REFERENCES}

[1] Human brain project - International neurionformatics research program[J]. Joumal of modem clinical medical, 2001,7(5):389-390

[2] Jin K, Mao XO, Sun Y, et al. Stem cell factor stimulates neurogenesis in vitro and in vivo[J]. J C lin Invest, 2002, 110(3): 311-319.

[3] Zhang SC, Fedoroff S. Expression of stem cell factor and ckit receptor in neural cells after brain injury [J]. A ctaNeuropathol (Berl), 1999, 97(4): 393-398.

[4] Ruggero Scorcioni, Sridevi Polavaram, Giorgio A Ascoli. L-Measure: a web-accessible tool for the analysis, comparison and search of digital reconstructions of neuronal morphologies. Nature Protocols 3,2008:866 - 876.

[5] http://neuromorpho.org/neuroMorpho/index.jsp.

[6] LUO Zhi-zeng, ZHAO Peng-fei. Nonlinear Principal Component Analysis for Feature Extraction of SEMG. Chinese journal of sensors and actuators, 2007, 20(10):2164-2168.

[7] SUN Jixiang. Modern Pattern Recognition [M].Changsha: National University of Defense Technology Press. 2002:285-288.

[8] Specht D. Probabilistic neural network[J]. Neural Networks, 1990,3(1):109-118.

[9] McDuff R J, Simpson P K. An Investigation of Neural Networks for F-16 Fault Dignosis:II. System Performance[A].SPIE 1294 Application of Artificial Neural Networks[C]. 1990:42-45.

[10] Liu Nangeng. Pattern recognition of SEMG based on prosthesis. A Dissertation to Shanghai Jiao Tong University for the Degree of Master. 2008 : 31-35.

[11] Erigui II, Krishnaparam R, Clustering by Competitive Agglomeration[J].PatternRecognition,1997,30(7):1109-1119.

[12] Avidan S.2005. Ensemble tracking. Proc. CVPR, 494-501.

[13] Grabner H, GRABNER M, Bischof H. 2006b. Real-time tracking via on-line boosting. Proc. British Machine Vision Conference,27-36.

[14] Hastie T, Tibshirani R, Friedman J. 2003. The Elements of Statistical Learning: Data Mining, Inference and Prediction. Springer.

[15] Huang C, Ai H, Li Y, et al. 2007. High-performance rotation invariant multiview face detection. IEEE Trans. PAMI,29(4) : 671-686. 